\title{
Bycatch fauna of seabob shrimp trawl fisheries from Santa Catarina State, southern Brazil
}

\author{
Joaquim Olinto Branco ${ }^{I}$, Felipe Freitas Júnior ${ }^{I}$ \& Martin Lindsey Christoffersen ${ }^{2,3}$ \\ ${ }^{1}$ Universidade do Vale do Itajaí, Centro de Ciências Tecnológicas da Terra e do Mar, Florianópolis, SC, \\ Brazil. \\ ${ }^{2}$ Universidade Federal da Paraíba, Departamento de Sistemática e Ecologia, João Pessoa, PB, Brazil. \\ ${ }^{3}$ Corresponding author: Martin Lindsey Christoffersen, e-mail: martinlc.ufpb@gmail.com
}

BRANCO, J.O., FREITAS JÚNIOR, F., CHRISTOFFERSEN, M.L. Bycatch fauna of seabob shrimp trawl fisheries from Santa Catarina State, southern Brazil. Biota Neotropica. 15(2): e20140143. http://dx.doi. org/10.1590/1676-06032015014314

\begin{abstract}
Marine communities in tropical regions contain a great diversity of species that play key roles in ecological processes. The observed bycatch fauna in artisanal fishing for the seabob shrimp Xiphopenaeus kroyeri (Heller, 1862) in the southwest Atlantic exceeds the quantity of commercial shrimp captured. This bycatch has not been previously inventoried extensively in Brazil, particularly in the State of Santa Catarina, where small-scale shrimp fisheries is a traditional activity and dominates the local fishing economy. Six municipalities were sampled three times a month, from 1996 to 2011, yielding 216 species belonging to the bycatch ( 7 cnidarians, 22 mollusks, 42 crustaceans, 11 echinoderms, and 134 fishes). Bycatch management is a pressing aspect of shrimp fisheries. By inventorying the accidental bycatch, we provide a preliminary step towards defining intervention measures to reduce or make better use of this bycatch in seabob artisanal fisheries. Conservation of marine biodiversity is still inadequate in Brazil. We have attempted to identify additional threats to vulnerable and endangered species resulting from shrimp trawling.

Keywords: bycatch fauna, marine diversity, southwest Atlantic, threatened species, Xiphopenaeus kroyeri.
\end{abstract}

BRANCO, J.O., FREITAS JÚNIOR, F., CHRISTOFFERSEN, M.L. Fauna acompanhante em pescas de arrastro do camarão sete-barbas em Santa Catarina, Brasil. Biota Neotropica. 15(2): e20140143. http:// dx.doi.org/10.1590/1676-06032015014314

Resumo: Comunidades marinhas em regiões tropicais contêm uma grande diversidade de espécies que desempenham papéis-chave em processos ecológicos. A fauna acompanhante em pescas artesanais do camarão sete-barbas Xiphopenaeus kroyeri (Heller, 1862) no Atlântico Sudoeste excede a quantidade de camarões comerciais desta espécie capturados. Esta fauna acompanhante não foi catalogada de forma detalhada no Brasil, particularmente no Estado de Santa Catarina, onde a pescaria em pequena escala é uma atividade tradicional que domina a economia pesqueira local. Seis municípios foram amostrados três vezes por mês, de 1996 a 2011, capturando 216 espécies pertencentes à fauna acompanhante (7 cnidários, 22 moluscos, 42 crustáceos, 11 equinodermos e 134 peixes). O manejo da fauna acompanhante constitui um aspecto urgente nas pescarias de camarões. Ao listar a fauna acompanhante acidental, uma etapa preliminar é executada para a definição de medidas de intervenção, fazendo melhor uso desta fauna acompanhante na pesca artesanal de camarões. A conservação da biodiversidade marinha ainda é inadequada no Brasil. Apresentamos uma tentativa de identificar ameaças adicionais às espécies vulneráveis e em risco de extinção como resultado das pescas de arrastro.

Palavras-chave: fauna acompanhante, diversidade marinha, Atlântico Sudoeste, espécies ameaçadas, Xiphopenaeus kroyeri.

\section{Introduction}

Biodiversity refers to the variety of life forms that result from the process of organic evolution, at all levels of organization. It may include intra and interspecific genetic variations or the diversity of ecosystems and the physical conditions under which they are found (Wilson 1992, Gray 1997, Alho 2008).

The importance of biodiversity for human life is presently being much discussed, either as a direct provider of natural products or as an indirect maintainer of ecological processes
(Alho 2008, Tundisi \& Matsumura-Tundisi 2008). One activity that benefits directly from biodiversity inventories is fisheries. Fishing stands out among the four largest producers of animal protein for human consumption in Brazil. According to data from FAO, the national production in shrimp fisheries occupied the 14th place in the world from 2000 to 2005 (Gillett 2008), while in 2006 Brazil became one of the six largest nations in terms of volume of shrimp fishery (Gillett 2008).

The small-scale fisheries of the seabob shrimp Xiphopenaeus kroyeri (Heller, 1862) exert a relevant role in the socioeconomic 
and cultural context along the coast of the State of Santa Catarina, Brazil. This activity represents a legacy of our Azorean culture, which contributed markedly to the development of the artisanal and industrial fisheries in the State (Branco 2005, Branco \& Verani 2006). On the other hand, due to the closing of net meshes during trawling, and thus to low selectivity of the fishing gear, a diversity of other organisms is captured incidentally. These organisms, the catch of individuals by fishing gear which is targeting another species (Keunecke et al. 2007), are known as the bycatch (Slavin 1983, Alverson et al. 1994).

This bycatch generally is quite diversified, consisting of fish, crustaceans, mollusks, among other groups. The bycatch is always considerably more abundant than the quantity of the commercial target shrimp (Coelho et al. 1986, Branco 2005). Unfortunately, shrimp trawling is undoubtedly the most damaging fishery activity in terms of biomass bycatch, and excessive bycatch discards certainly occur in Brazilian fisheries (Viana \& Almeida 2005). Part of this capture, represented by specimens of economic importance and sufficient commercial size is landed, while another part, frequently the largest fraction, composed of individuals with no commercial value or by commercial specimens of small size, is returned non-living to the sea (Graça-Lopes et al. 2002, Branco 2005). This fact, allied with overfishing and the pollution of oceans, is being pinpointed as one of the main threats to the biodiversity of the marine environment (Amaral \& Jablonski 2005). As an aggravating factor, tropical shrimp fishery has been found to generate the highest proportion of discard in relation to other fishery activities (Alverson et al.1994). In Brazil, studies on the biology of discarded species are deficient and scarce (Vianna et al. 2000). Such studies increase in importance when many fishery resources show signs of depletion (Vianna \& Verani 2002). Vianna \& D' Incao (2006) demonstrated that the use of a simple bycatch reduction device grid will greatly reduce the negative impact of this bycatch waste. Other papers (Vianna 2001, Vianna et al. 2004, Vianna \& Almeida 2005, Keunecke et al. 2007) further study the impact of shrimp fisheries on the bycatch fauna.

Notwithstanding these threats, little is known about marine biodiversity (Hendrickx et al. 2002, Amaral \& Jablonski 2005, Severino-Rodrigues et al. 2007, Perez 2009), especially regarding shrimp fisheries (Graça-Lopes et al. 2002, Pinheiro \& Martins 2009). Bycatch is considered in Vianna (2001), Vianna et al. (2004), Keunecke et al. (2007) and Vianna \& Almeida (2009). Yet most papers in our country deal only with a few individual groups, such as the ichthyofauna of Pernambuco and Alagoas (Santos et al. 2008, Santos 2000, Tischer \& Santos 2001), São Paulo (Graça Lopes et al. 2002, Coelho et al. 1986, Paiva-Filho \& Schmiegelow 1986), Paraná (Gomes \& Chaves 2006, Schwarz Jr et al. 2007), Santa Catarina (Branco \& Verani 2006, Bernardes Jr et al. 2011; Freitas et al. 2011), or the carcinofauna inventoried by Severino-Rodrigues et al. (2002), Robert et al. (2007) and Branco \& Fracasso (2004), respectively, in São Paulo, Paraná and Santa Catarina.

In Santa Catarina, the analysis of the rejected bycatch is still deficient. This paper thus aims to characterize for the first time the species composition associated with the seabob shrimp fisheries, in six municipalities of the State, as a subsidy for evaluating the impact of this fishing gear in the coastal environment.

\section{Material and methods}

The present study was conducted along the coast of the State of Santa Catarina, southern Brazil, in the main fishing

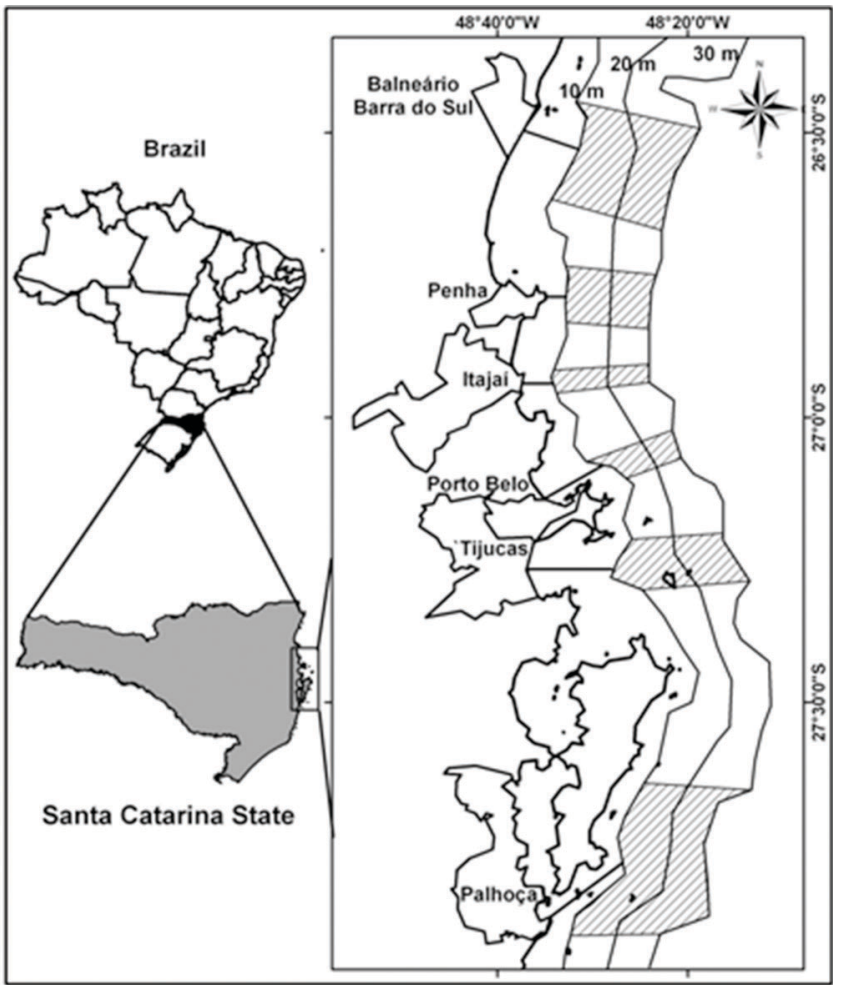

Figure 1. On the left the insertion of the State of Catarina in southern Brazil is indicated. On the right the entire coastline of the State informs the names of the coastal municipalities from north to south and shows the shallow coastal area down to 30 meters depth, the longitudinal lines indicating the isobaths of 10 and $20 \mathrm{~m}$. The hatched areas represent the sampled fishing areas.

areas of the seabob shrimp, between the coordinates of $26^{\circ} 24^{\prime}-27^{\circ} 53^{\prime} \mathrm{S}$ and $48^{\circ} 33^{\prime}-48^{\circ} 38^{\prime} \mathrm{W}$, covering the municipalities of Barra do Sul, Penha, Itajaí, Porto Belo, Tijucas, and Palhoça (Fig. 1, Table 1).

In each locality three trawls per month were made, in traditional fishing sites, with a mean duration of one hour, in depths varying from 5 to $30 \mathrm{~m}$, from 1996 to 2011. For the capture of specimens, a vessel was equipped with two doublerigged trawl nets, with net meshes of $3.0 \mathrm{~cm}$ at net entrance and $2.0 \mathrm{~cm}$ in the collecting sac, and trawled at a mean speed of two knots (Branco 2005). The contents of each trawling were conditioned, tagged and maintained in isoprene boxes with ice.

In the lab, identification of the components of the fauna was made with specialized references for each group. Families were listed in conventional taxonomical order, species in alphabetical order. Species were grouped as very frequent (VF), when occurring in more than $70 \%$ of the samples; frequent $(\mathrm{F})$, when abundance is from $40 \%$ to $69,9 \%$ of the samples; little frequent (LF), when abundance is between $20 \%$ and $39,9 \%$; and occasional $(\mathrm{O})$, when occurring in less than $19,9 \%$ of the samples (Severino-Rodrigues et al. 2002).

\section{Results}

During the study period a total of 216 species were identified in the artisanal fisheries for the seabob shrimp along the coast of the State of Santa Catarina, belonging to 105 families, among cnidarians, mollusks, crustaceans, echinoderms and fishes (Tables 2 to 6). 
Table 1. Number of tows and depths ranges at each collecting site.

\begin{tabular}{llll}
\hline Municipality & Years & Number of tows & Depth range (m) \\
\hline Barra do Sul & $2007-2010$ & 144 & $10-20-30$ \\
Penha & $1996-2011$ & 540 & $10-20-30$ \\
Itajaí & $2001-2002$ & 72 & $05-10-20$ \\
Porto Belo & $2009-2010$ & 72 & $10-20-30$ \\
Tijucas & $2004-2005,2008$ & 108 & $08-15-20$ \\
Palhoça & $2003-2004$ & 72 & $10-20-30$ \\
\hline
\end{tabular}

Table 2. List of cnidarian species, with respective frequencies of occurrence by collecting site, during the period between years 1996 and 2010 . VF: very frequent; F: frequent; LF: little frequent; O: occasional.

\begin{tabular}{|c|c|c|c|c|c|c|}
\hline Family/Species & Barra do Sul & Penha & Itajaí & Tijucas & Porto Belo & Palhoça \\
\hline \multicolumn{7}{|l|}{ Cnidaria/ Anthozoa } \\
\hline $\begin{array}{l}\text { Bunodosoma caissarum Corrêa, } 1964 \\
\text { Renillidae }\end{array}$ & VF & VF & $\mathrm{F}$ & $\mathrm{F}$ & VF & $\mathrm{F}$ \\
\hline $\begin{array}{l}\text { Renilla muelleri Kölliker, } 1872 \\
\text { Cnidaria/ Hydrozoa }\end{array}$ & $\mathrm{O}$ & $\mathrm{O}$ & & & $\mathrm{O}$ & $\mathrm{O}$ \\
\hline $\begin{array}{l}\text { Olindiidae } \\
\text { Olindias sambaquiensis Müller, } 1861\end{array}$ & $\mathrm{O}$ & $\mathrm{O}$ & $\mathrm{O}$ & $\mathrm{O}$ & $\mathrm{O}$ & $\mathrm{O}$ \\
\hline $\begin{array}{l}\text { Aequoridae } \\
\text { Rhacostoma atlantica Agassiz, } 1850 \\
\text { Cnidaria/ Cubozoa }\end{array}$ & $\mathrm{F}$ & $\mathrm{F}$ & $\mathrm{F}$ & $\mathrm{F}$ & $\mathrm{F}$ & $\mathrm{F}$ \\
\hline $\begin{array}{l}\text { Chirodropidae } \\
\text { Chiropsalmus quadrumanus (Müller , 1859) } \\
\text { Cnidaria/ Scyphozoa }\end{array}$ & VF & VF & VF & $\mathrm{VF}$ & $\mathrm{VF}$ & VF \\
\hline $\begin{array}{l}\text { Chrysaora lactea } \text { Eschscholtz, } 1829 \\
\text { Lychnorhizidae }\end{array}$ & $\mathrm{O}$ & $\mathrm{O}$ & $\mathrm{O}$ & $\mathrm{O}$ & $\mathrm{O}$ & $\mathrm{O}$ \\
\hline Lychnorhiza lucerna Haeckel, 1880 & $\mathrm{~F}$ & $\mathrm{~F}$ & $\mathrm{~F}$ & $\mathrm{LF}$ & $\mathrm{F}$ & $\mathrm{F}$ \\
\hline
\end{tabular}

Cnidarians were represented by seven species, in seven genera and families, belonging to the classes Anthozoa, Hydrozoa, Cubozoa, and Scyphozoa. The anemone Bunodosoma caissarum and the medusa Chiropsalmus quadrumanus were considered very frequent or frequent in all sampled areas, while the penatulacean Renilla muelleri and the medusae Chrysaora lactea and Olindias sambaquiensis occurred occasionally (Table 2). Rhacostoma atlanticum and Lychnorhiza lucerna were frequent in the six regions, except in Tijucas, where L. lucerna was recorded with low frequency (Table 2).

Mollusks participated with 22 species, 21 genera and 19 families, distributed among Gastropoda, Bivalva and Cephalopoda (Table 3). Gastropods contributed with the largest number of families and species, of which 11 species occurred occasionally, while Buccinanops gradatum was very frequent in most sampled regions ( 10 were recorded in Table 3 ), followed by Olivancillaria urceus. The only captured polyplacophoran, Chaetopleura angulata, appeared occasionally (Table 3).

Six species of Bivalvia were recorded only in the region of Penha, occurring occasionally in samples (Table 3). Of the four Cephalopoda, the squid Lolliguncula brevis was very frequent (except in Itajaí), Loligo plei and Loligo sanpaulensis were frequent in Tijucas and Porto Belo, very frequent in Palhoça and occasional/little frequent in the remaining areas. Octopus vulgaris appeared occasionally in most areas, except in Itajaí and Tijucas (Table 3).

Among crustaceans, 42 species were identified (including the seabob shrimp), distributed into 33 genera and 17 families. Stomatopoda were represented by one family (Squillidae) and species (Squilla empusa), occurring little frequently or occasionally in trawls (Table 4). Decapoda contributed with a larger number of species. Portunidae with 9 species, Callinectes danae and $C$. ornatus being the most frequent, followed by Xanthidae and Penaeidae, both with six species (Table 4).

Hepatus pudibundus was the most frequent crab in captures, while Leucosiidae, Majidae and Parthenopidae oscillated between little frequent and occasional in the fishing areas (Table 4).

Among the Xanthidae, all species were occasional, while Penaeidae, represented by the marine shrimps of commercial value, had only the seabob shrimp (Xiphopenaeus kroyeri) as very frequent, followed by Artemesia longinaris, frequent in all localities (Table 4). The pink-shrimp (Farfantepenaeus brasiliensis and $F$. paulensis) and the white shrimp (Litopenaeus schmitti) alternated between little frequent and occasional in most sampled localities, except in Itajaí, where L. schmitti was frequent and in Palhoça, where $F$. brasiliensis was frequent (Table 4). 
Table 3. List of mollusk species, with respective frequencies of occurrence by collecting site, during the period between years 1996 and 2010 . VF: very frequent; F: frequent; LF: little frequent; O: occasional.

\begin{tabular}{|c|c|c|c|c|c|c|}
\hline Family/Species & Barra do Sul & Penha & Itajaí & Tijucas & Porto Belo & Palhoça \\
\hline \multicolumn{7}{|l|}{ Mollusca/ Gastropoda } \\
\hline \multicolumn{7}{|l|}{ Trochidae } \\
\hline Tegula viridula (Gmelin, 1791) & & $\mathrm{O}$ & & & & LF \\
\hline \multicolumn{7}{|l|}{ Turbinidae } \\
\hline Astraea latispina (Philippi, 1844) & & $\mathrm{O}$ & & & & \\
\hline \multicolumn{7}{|l|}{ Tonnidae } \\
\hline Tonna galea brasiliana (Mörch, 1877) & & $\mathrm{O}$ & & & & \\
\hline \multicolumn{7}{|l|}{ Ranellidae } \\
\hline Cymatium parthenopeum (von Salis, 1793) & $\mathrm{O}$ & $\mathrm{O}$ & $\mathrm{O}$ & $\mathrm{O}$ & $\mathrm{O}$ & $\mathrm{O}$ \\
\hline \multicolumn{7}{|l|}{ Muricidae } \\
\hline Siratus senegalensis (Gmelin, 1790) & $\mathrm{O}$ & $\mathrm{O}$ & $\mathrm{O}$ & $\mathrm{O}$ & $\mathrm{O}$ & \\
\hline \multicolumn{7}{|l|}{ Thaididae } \\
\hline Thais haemastoma (Linnaeus, 1767) & & $\mathrm{O}$ & & & & $\mathrm{O}$ \\
\hline \multicolumn{7}{|l|}{ Nassariidae } \\
\hline Buccinanops gradatum (Deshayes, 1844) & $\mathrm{VF}$ & $\mathrm{VF}$ & $\mathrm{F}$ & VF & VF & $\mathrm{F}$ \\
\hline \multicolumn{7}{|l|}{ Strombidae } \\
\hline Strombus pugilis (Linnaeus, 1758) & & $\mathrm{O}$ & & & & \\
\hline \multicolumn{7}{|l|}{ 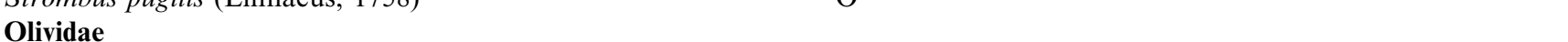 } \\
\hline Olivancillaria urceus (Röding, 1798) & $\mathrm{VF}$ & $\mathrm{VF}$ & $\mathrm{F}$ & $\mathrm{F}$ & $\mathrm{F}$ & $\mathrm{F}$ \\
\hline \multicolumn{7}{|l|}{ Volutidae } \\
\hline Zidona dufresnei (Donovan, 1823) & $\mathrm{O}$ & $\mathrm{O}$ & & & $\mathrm{O}$ & \\
\hline \multicolumn{7}{|l|}{ Aplysiidae } \\
\hline Aplysia brasiliana Rang, 1828 & $\mathrm{O}$ & $\mathrm{O}$ & & & & \\
\hline \multicolumn{7}{|l|}{ Mollusca/ Polyplacophora } \\
\hline \multicolumn{7}{|l|}{ Ischnochitonidae } \\
\hline Chaetopleura angulata (Spengler, 1797) & $\mathrm{O}$ & $\mathrm{O}$ & & & $\mathrm{O}$ & $\mathrm{O}$ \\
\hline \multicolumn{7}{|l|}{ Mollusca/ Bivalvia } \\
\hline \multicolumn{7}{|l|}{ Arcidae } \\
\hline Anadara brasiliana (Lamarck, 1819) & & $\mathrm{O}$ & & $\mathrm{O}$ & & \\
\hline \multicolumn{7}{|l|}{ Mvtilidae } \\
\hline \multirow{2}{*}{\multicolumn{7}{|c|}{$\begin{array}{l}\text { Modiolus carvalhoi Klappenbach, } 1966 \\
\text { Cardiidae }\end{array}$}} \\
\hline & & & & & & \\
\hline Trachycardium muricatum (Linnaeus, 1758) & & $\mathrm{O}$ & & & & \\
\hline \multicolumn{7}{|l|}{ Veneridae } \\
\hline Chione cancellata (Linnaeus, 1767) & & $\mathrm{O}$ & & & & \\
\hline \multirow{2}{*}{\multicolumn{7}{|c|}{$\begin{array}{l}\text { Pltar arestus (Dall and simpson, 1901) } \\
\text { Tellinide }\end{array}$}} \\
\hline & & & & & & \\
\hline Temnoconcha brasiliana Dall, 1921 & & $\mathrm{O}$ & & & & \\
\hline \multicolumn{6}{|l|}{ Mollusca/ Cephalopoda } & \\
\hline \multicolumn{7}{|l|}{ Loliginidae } \\
\hline Loligo plei Blainville, 1823 & LF & LF & $\mathrm{O}$ & $\mathrm{F}$ & $\mathrm{F}$ & $\mathrm{VF}$ \\
\hline Loligo sanpaulensis Brakoniecki, 1984 & $\mathrm{O}$ & LF & LF & $\mathrm{F}$ & $\mathrm{F}$ & VF \\
\hline Lolliguncula brevis (Blainville, 1823) & VF & $\mathrm{VF}$ & $\mathrm{F}$ & $\mathrm{VF}$ & VF & $\mathrm{VF}$ \\
\hline \multicolumn{7}{|l|}{ Otopodidae } \\
\hline Otopus vulgaris Cuvier, 1797 & $\mathrm{O}$ & $\mathrm{O}$ & & & $\mathrm{O}$ & $\mathrm{O}$ \\
\hline
\end{tabular}

The solenocerid Pleoticus muelleri, has commercial importance in Santa Catarina, being frequent from Barra do Sul to Tijucas and very frequent in Porto Belo and Palhoça (Table 4). In the latter location, this species, together with Artemesia longinaris, contributed with the largest abundances, superseding the target species of shrimp. The shrimp Acetes americanus (Sergestidae) also had high frequency in captures, alternating between frequent and very frequent (Table 4).

Among hermit crabs (here represented by families Diogenidae and Paguridae), only Dardanus insignis had a high frequency of occurrence, being very frequent in Barra do Sul - Penha and frequent in the remaining localities. The remaining species have occasional or little frequent occurrences (Table 4). The crab-like Porcellana sayana was the most frequent anomuran in captures (Table 4).

The echinoderms were represented by 11 species, distributed in nine genera and families, among Asteroidea, Echinoidea and Ophiuroidea, where the first contributed with the largest number of families and species, with special mention of the sea-star Astropecten marginatus. Although this latter 
Table 4. List of crustacean species, with respective frequencies of occurrence by collecting site, during the period between years 1996 and 2010 . VF: very frequent; F: frequent; LF: little frequent; O: occasional.

\begin{tabular}{|c|c|c|c|c|c|c|}
\hline Family/Species & Barra do Sul & Penha & Itajaí & Tijucas & Porto Belo & Palhoça \\
\hline \multicolumn{7}{|l|}{ Squillidae } \\
\hline Squilla empusa Say, 1818 & LF & LF & & $\mathrm{O}$ & $\mathrm{LF}$ & LF \\
\hline \multicolumn{7}{|l|}{ Crustaceal Decapoda } \\
\hline \multicolumn{7}{|l|}{ Penaeidae } \\
\hline Artemesia longinaris Bate, 1888 & $\mathrm{~F}$ & $\mathrm{~F}$ & $\mathrm{~F}$ & $\mathrm{~F}$ & $\mathrm{~F}$ & $\mathrm{~F}$ \\
\hline Farfantepenaeus brasiliensis (Latreille, 1817) & LF & $\mathrm{O}$ & $\mathrm{LF}$ & $\mathrm{O}$ & LF & $\mathrm{F}$ \\
\hline Farfantepenaeus paulensis (Pérez Farfante, 1967) & $\mathrm{O}$ & $\mathrm{O}$ & LF & $\mathrm{O}$ & $\mathrm{O}$ & $\mathrm{LF}$ \\
\hline Litopenaeus schmitti (Burkenroad, 1936) & LF & LF & $\mathrm{F}$ & LF & $\mathrm{LF}$ & $\mathrm{LF}$ \\
\hline Rimapenaeus constrictus (Stimpson, 1871) & $\mathrm{O}$ & $\mathrm{O}$ & $\mathrm{O}$ & $\mathrm{O}$ & $\mathrm{LF}$ & $\mathrm{O}$ \\
\hline Xiphopenaeus kroyeri (C. Heller, 1862) & VF & $\mathrm{VF}$ & VF & VF & VF & $\mathrm{LF}$ \\
\hline \multicolumn{7}{|l|}{ Solenoceridae } \\
\hline Pleoticus muelleri (Bate, 1888) & $\mathrm{F}$ & $\mathrm{F}$ & $\mathrm{F}$ & $\mathrm{F}$ & $\mathrm{VF}$ & VF \\
\hline \multicolumn{7}{|l|}{ Sicyoniidae } \\
\hline Sicyonia dorsalis Kingsley, 1878 & $\mathrm{~F}$ & $\mathrm{~F}$ & LF & $\mathrm{F}$ & VF & LF \\
\hline \multicolumn{7}{|l|}{ Alpheidae } \\
\hline Alpheus bouvieri A. Milne-Edwards, 1878 & & LF & $\mathrm{O}$ & & $\mathrm{O}$ & $\mathrm{O}$ \\
\hline \multicolumn{7}{|l|}{ Lysmatidae } \\
\hline Exhippolysmata oplophoroides (Holthuis, 1948) & $\mathrm{O}$ & LF & $\mathrm{O}$ & LF & $\mathrm{LF}$ & $\mathrm{O}$ \\
\hline \multicolumn{7}{|l|}{ Sergestidae } \\
\hline Acetes americanus Ortmann, 1893 & VF & VF & VF & $\mathrm{F}$ & $\mathrm{F}$ & $\mathrm{F}$ \\
\hline \multicolumn{7}{|l|}{ Palaemonidae } \\
\hline Nematopalaemon schmitti (Holthuis,1950) & & $\mathrm{O}$ & $\mathrm{O}$ & & & \\
\hline \multicolumn{7}{|l|}{ Diogenidae } \\
\hline Dardanus insignis (de Saussure, 1858) & VF & VF & $\mathrm{F}$ & $\mathrm{F}$ & $\mathrm{F}$ & $\mathrm{F}$ \\
\hline Dardanus venosus (H. Milne Edwards, 1848) & & $\mathrm{O}$ & & & $\mathrm{O}$ & \\
\hline Petrochirus diogenes (Linnaeus, 1758) & $\mathrm{O}$ & $\mathrm{O}$ & $\mathrm{O}$ & $\mathrm{O}$ & $\mathrm{O}$ & $\mathrm{O}$ \\
\hline Loxopagurus loxochelis (Moreira, 1901) & LF & LF & LF & LF & $\mathrm{LF}$ & $\mathrm{O}$ \\
\hline \multicolumn{7}{|l|}{ Paguridae } \\
\hline Pagurus exilis (Benedict, 1892) & & $\mathrm{O}$ & & & $\mathrm{O}$ & \\
\hline \multicolumn{7}{|l|}{ Porcellanidae } \\
\hline Porcellana sayana (Leach, 1820) & $\mathrm{VF}$ & $\mathrm{VF}$ & $\mathrm{F}$ & $\mathrm{F}$ & $\mathrm{F}$ & $\mathrm{LF}$ \\
\hline \multicolumn{7}{|l|}{ Aethridae } \\
\hline Hepatus pudibundus (Herbst, 1785) & $\mathrm{VF}$ & $\mathrm{VF}$ & $\mathrm{VF}$ & $\mathrm{F}$ & VF & VF \\
\hline \multicolumn{7}{|l|}{ Leucosiidae } \\
\hline Persephona lichtensteinii Leach, 1817 & $\mathrm{O}$ & LF & LF & $\mathrm{O}$ & $\mathrm{F}$ & LF \\
\hline Persephona mediterranea (Herbst, 1794) & $\mathrm{F}$ & LF & $\mathrm{O}$ & $\mathrm{O}$ & $\mathrm{F}$ & $\mathrm{LF}$ \\
\hline Persephona punctata (Linnaeus, 1758) & LF & LF & LF & $\mathrm{O}$ & $\mathrm{LF}$ & $\mathrm{LF}$ \\
\hline \multicolumn{7}{|l|}{ Epialtidae } \\
\hline Libinia ferreirae Brito Capello, 1871 & $\mathrm{O}$ & & & & $\mathrm{O}$ & \\
\hline Libinia spinosa (H. Milne Edwards, 1834) & $\mathrm{O}$ & LF & LF & $\mathrm{O}$ & $\mathrm{F}$ & LF \\
\hline Pelia rotunda A. Milne-Edwards, 1875 & & $\mathrm{O}$ & & & & \\
\hline \multicolumn{7}{|l|}{ Majidae } \\
\hline Microphrys bicornutus (Latreille, 1825) & & $\mathrm{O}$ & & & & \\
\hline \multicolumn{7}{|l|}{ Parthenopidae } \\
\hline Heterocrypta lapidea Rathbun, 190 & & $\mathrm{O}$ & & & & \\
\hline Polybiidae & & & & & & \\
\hline Ovalipes trimaculatus (De Haan 1833) & & & & & & $\mathrm{LF}$ \\
\hline Portunidae & & & & & & \\
\hline Arenaeus cribrarius (Lamarck, 1818) & LF & LF & LF & & $\mathrm{F}$ & $\mathrm{O}$ \\
\hline Callinectes danae Smith, 1869 & VF & VF & VF & VF & VF & $\mathrm{F}$ \\
\hline Callinectes bocourti A Milne Edwards, 1879 & & & $\mathrm{O}$ & & & \\
\hline Callinectes ornatus Ordway, 1863 & $\mathrm{VF}$ & $\mathrm{VF}$ & $\mathrm{VF}$ & $\mathrm{VF}$ & VF & VF \\
\hline Callinectes sapidus Rathbun, 1896 & & $\mathrm{O}$ & $\mathrm{O}$ & $\mathrm{O}$ & & \\
\hline Cronius ruber (Lamarck, 1818) & & $\mathrm{O}$ & & & $\mathrm{O}$ & $\mathrm{O}$ \\
\hline Portunus spinicarpus (Stimpson, 1871) & $\mathrm{O}$ & $\mathrm{O}$ & & & $\mathrm{LF}$ & $\mathrm{LF}$ \\
\hline Achelous spinimanus (Latreille, 1819) & LF & $\mathrm{O}$ & $\mathrm{O}$ & & $\mathrm{F}$ & LF \\
\hline
\end{tabular}


Branco, J.O. et al.

Table 4. Continued.

\begin{tabular}{|c|c|c|c|c|c|c|}
\hline Family/Species & Barra do Sul & Penha & Itajaí & Tijucas & Porto Belo & Palhoça \\
\hline \multicolumn{7}{|l|}{ Panopeidae } \\
\hline Acantholobulus schmitti (Rathbun, 1930) & & $\mathrm{O}$ & & & & \\
\hline Eurypanopeus abbreviatus (Stimpson, 1860) & & $\mathrm{O}$ & & & & \\
\hline \multicolumn{7}{|l|}{ Menippidae } \\
\hline Menippe nodifrons Stimpson, 1859 & $\mathrm{O}$ & $\mathrm{O}$ & & & $\mathrm{O}$ & $\mathrm{O}$ \\
\hline \multicolumn{7}{|l|}{ Pilumnidae } \\
\hline Pilumnus dasypodus Kingsley, 1879 & $\mathrm{O}$ & $\mathrm{O}$ & $\mathrm{O}$ & $\mathrm{O}$ & $\mathrm{O}$ & $\mathrm{O}$ \\
\hline \multicolumn{7}{|l|}{ Xanthidae } \\
\hline Speocarcinus carolinensis Stimpson, 1859 & & $\mathrm{O}$ & & & $\mathrm{O}$ & \\
\hline Speocarcinus meloi D'incao and Gomes da Silva, 1992 & & & & & $\mathrm{O}$ & \\
\hline
\end{tabular}

Table 5. List of echinoderm species, with respective frequencies of occurrence by collecting site, during the period between years 1996 and 2010 . VF: very frequent; F: frequent; LF: little frequent; O: occasional.

\begin{tabular}{|c|c|c|c|c|c|c|}
\hline Family/Species & Barra do Sul & Penha & Itajaí & Tijucas & Porto Belo & Palhoça \\
\hline \multicolumn{7}{|l|}{ Echinodermata/ Asteroidea } \\
\hline Astropecten brasiliensis Müller and Troschel, 1842 & $\mathrm{O}$ & $\mathrm{O}$ & $\mathrm{O}$ & & LF & LF \\
\hline Astropecten marginatus (Gray, 1840) & $\mathrm{F}$ & VF & VF & $\mathrm{F}$ & $\mathrm{VF}$ & $\mathrm{VF}$ \\
\hline \multicolumn{7}{|l|}{ Luidiidae } \\
\hline Luidia clathrata (Say, 1825) & & $\mathrm{O}$ & $\mathrm{O}$ & & & \\
\hline \multicolumn{7}{|l|}{ Asterinidae } \\
\hline \multicolumn{7}{|l|}{ Echinasteridae } \\
\hline Echinaster brasiliensis (Müller and Troschel, 1842) & & $\mathrm{O}$ & & & $\mathrm{O}$ & $\mathrm{O}$ \\
\hline \multicolumn{7}{|l|}{ Echinodermata/ Echinoidea } \\
\hline \multicolumn{7}{|l|}{ Mellitidae } \\
\hline \multicolumn{7}{|l|}{ Toxopneustidae } \\
\hline $\begin{array}{l}\text { Lytechinus variegatus (Lamarck, 1816) } \\
\text { Echinodermata/Ophiuroidea } \\
\text { Amphiuridae }\end{array}$ & & $\mathrm{O}$ & & & $\mathrm{O}$ & LF \\
\hline \multicolumn{6}{|l|}{ Ophiactidae } & $\mathrm{O}$ \\
\hline Hemipholis elongata (Say, 1825) & $\mathrm{O}$ & $\mathrm{O}$ & & & & $\mathrm{O}$ \\
\hline
\end{tabular}

species is considered threatened, it was found very frequently in most sampled localities (Table 5). The remaining species of Asteroidea occurred occasionally or with little frequency, such as Luidia senegalensis (Table 5).

Among the Echinoidea, Arbacia lixula, Lytechinus variegatus and Mellita quinquiesperforata were registered occasionally in Penha and Porto Belo, and frequently ( $M$. quinquiesperforata), or little frequently (L. variegatus) in Palhoça (Table 5). Ophiuroidea, with Micropholis atra and Hemipholis elongata, were of occasional occurrences in Penha, Barra do Sul and Palhoça (Table 5).

Of all the faunistic groups captured with the seabob shrimp, the ichthyofauna presented the largest number of families and species, being represented by 134 species, distributed in 53 families. Ten were elasmobranchs and 124 were bony fishes (Table 6). Elasmobranchs had a small presence in the trawls, occurring occasionally in samples, with the exception of the rays Zapteryx brevirostris and Atlantoroja cyclophora, frequent in the fisheries at Porto Belo (Table 6).

The 124 species of bony fish are distributed into 92 genera and 48 families, the occasional and little frequent species dominating the captures (Table 6). Sciaenidae contributed with most species, with high frequencies of occurrence, mainly Paralonchurus brasiliensis, Stellifer brasiliensis and $S$. rastrifer, which were very frequent in all sampled areas. They were followed by Isopisthus parvipinnis, Menticirrhus americanus, Stellifer stellifer and Larimus breviceps, which oscillated from frequent to very frequent (except for Tijucas and Palhoça) (Table 6). Micropogonias furnieri presented high frequency in Itajaí and Porto Belo, Cynoscion jamaicensis and Cynoscion guatacupa in Porto Belo and Palhoça, respectively, and Ctenosciaena gracilicirrhus in Penha.

The Sciaenidae presented the highest specific diversity, 16 species recorded, followed by 12 species of Carangidae and 
Table 6. List of fish species, with respective frequencies of occurrence by collecting site, during the period between years 1996 and 2010 . VF: very frequent; F: frequent; LF: little frequent; O: occasional; *, threatened species.

\begin{tabular}{|c|c|c|c|c|c|c|}
\hline Family/Species & Barra do Sul & Penha & Itajaí & Tijucas & Porto Belo & Palhoça \\
\hline \multicolumn{7}{|l|}{ Chondrichthyes } \\
\hline \multicolumn{7}{|l|}{ Squatinidae } \\
\hline Squatina guggenheim Marini, $1936^{*}$ & & $\mathrm{O}$ & & & & \\
\hline \multicolumn{7}{|l|}{ Narcinidae } \\
\hline Narcine brasiliensis (Olfers, 1831)* & $\mathrm{O}$ & $\mathrm{O}$ & $\mathrm{O}$ & $\mathrm{O}$ & & $\mathrm{O}$ \\
\hline \multicolumn{7}{|l|}{ Rhinobatidae } \\
\hline Rhinobatos horkelii Müller and Henle, 1841 & $\mathrm{O}$ & $\mathrm{O}$ & & & & \\
\hline Rhinobatos percellens (Walbaum, 1792) & & $\mathrm{O}$ & & & & $\mathrm{O}$ \\
\hline Zapteryx brevirostris (Müller and Henle, 1841) & LF & $\mathrm{O}$ & $\mathrm{O}$ & & $\mathrm{F}$ & $\mathrm{O}$ \\
\hline \multicolumn{7}{|l|}{ Rajidae } \\
\hline Atlantoraja cyclophora (Regan, 1903) & $\mathrm{O}$ & $\mathrm{O}$ & $\mathrm{O}$ & & $\mathrm{F}$ & $\mathrm{O}$ \\
\hline Rioraja agassizi (Müller and Henle, 1841) & $\mathrm{O}$ & $\mathrm{O}$ & $\mathrm{O}$ & & $\mathrm{LF}$ & $\mathrm{O}$ \\
\hline Sympterygia acuta Garman, 1877 & & & & & & $\mathrm{O}$ \\
\hline Sympterygia bonapartei Muller and Henle, 1841 & & & & & & $\mathrm{O}$ \\
\hline \multicolumn{7}{|l|}{ Rhinopteridae } \\
\hline Rhinoptera bonasus (Mitchill, 1815) & & & & & $\mathrm{O}$ & \\
\hline \multicolumn{7}{|l|}{ Actinopterigii } \\
\hline \multicolumn{7}{|l|}{ Elopidae } \\
\hline Elops saurus Linnaeus, 1776 & & $\mathrm{O}$ & $\mathrm{O}$ & $\mathrm{O}$ & & \\
\hline \multicolumn{7}{|l|}{ Muraenidae } \\
\hline Gymnothorax ocellatus (Agassiz, 1831) & $\mathrm{O}$ & LF & & & $\mathrm{F}$ & LF \\
\hline \multicolumn{7}{|l|}{ Ophichthidae } \\
\hline Ophichthus gomesii (Castelnau, 1855) & $\mathrm{O}$ & LF & $\mathrm{O}$ & LF & $\mathrm{O}$ & $\mathrm{O}$ \\
\hline \multicolumn{7}{|l|}{ Muraenesidae } \\
\hline Cynoponticus savanna (Bancroft, 1831) & & & & & & $\mathrm{O}$ \\
\hline \multicolumn{7}{|l|}{ Congridae } \\
\hline Conger orbignianus Valenciennes, 1837 & $\mathrm{O}$ & $\mathrm{O}$ & & $\mathrm{O}$ & $\mathrm{LF}$ & $\mathrm{O}$ \\
\hline \multicolumn{7}{|l|}{ Pristigasteridae } \\
\hline Chirocentrodon bleekerianus (Poey, 1867) & VF & $\mathrm{F}$ & $\mathrm{O}$ & $\mathrm{O}$ & $\mathrm{LF}$ & $\mathrm{O}$ \\
\hline Pellona harroweri (Fowler, 1917) & VF & $\mathrm{F}$ & $\mathrm{F}$ & VF & $\mathrm{VF}$ & LF \\
\hline \multicolumn{7}{|l|}{ Engraulidae } \\
\hline Anchoa spinifer (Valenciennes, 1848) & $\mathrm{O}$ & $\mathrm{O}$ & $\mathrm{O}$ & & & $\mathrm{O}$ \\
\hline Anchoviella lepidentostole (Fowler, 1911) & $\mathrm{O}$ & LF & $\mathrm{O}$ & $\mathrm{O}$ & $\mathrm{O}$ & \\
\hline Cetengraulis edentulus (Cuvier,1829) & & $\mathrm{O}$ & LF & $\mathrm{O}$ & & \\
\hline Lycengraulis grossidens Agassiz, 1829 & $\mathrm{O}$ & LF & LF & $\mathrm{O}$ & $\mathrm{O}$ & $\mathrm{O}$ \\
\hline \multicolumn{7}{|l|}{ Clupeidae } \\
\hline Harengula clupeola (Cuvier, 1829) & $\mathrm{O}$ & LF & LF & $\mathrm{O}$ & $\mathrm{O}$ & $\mathrm{O}$ \\
\hline Opisthonema oglinum (Lesuer, 1818) & $\mathrm{O}$ & $\mathrm{O}$ & $\mathrm{O}$ & $\mathrm{O}$ & $\mathrm{O}$ & $\mathrm{O}$ \\
\hline Sardinella brasiliensis (Steindachner, 1879) & $\mathrm{O}$ & $\mathrm{O}$ & $\mathrm{O}$ & $\mathrm{O}$ & $\mathrm{O}$ & $\mathrm{O}$ \\
\hline \multicolumn{7}{|l|}{ Ariidae } \\
\hline Cathorops spixii (Agassiz, 1829) & & $\mathrm{O}$ & & VF & & \\
\hline Genidens genidens (Valenciennes, 1839) & $\mathrm{O}$ & LF & $\mathrm{F}$ & $\mathrm{F}$ & $\mathrm{LF}$ & $\mathrm{O}$ \\
\hline Genidens barbus (Lacépedède, 1803) & $\mathrm{O}$ & LF & LF & $\mathrm{F}$ & $\mathrm{F}$ & LF \\
\hline \multicolumn{7}{|l|}{ Synodontidae } \\
\hline Synodus foetens (Linnaeus, 1766) & $\mathrm{LF}$ & & & & & LF \\
\hline Synodus intermedius (Spix and Agassiz, 1829) & $\mathrm{O}$ & & & & & $\mathrm{O}$ \\
\hline TrachinOephalus myops (Forster, 1801) & $\mathrm{F}$ & & & & & \\
\hline Phycidae & & & & & & \\
\hline Urophycis brasiliensis (Kaup, 1858) & $\mathrm{O}$ & $\mathrm{O}$ & LF & & $\mathrm{F}$ & LF \\
\hline Ophidiidae & & & & & & \\
\hline Ophidion holbrookii Putnam, 1874 & $\mathrm{O}$ & $\mathrm{O}$ & $\mathrm{O}$ & & & $\mathrm{O}$ \\
\hline Raneya brasiliensis (Kaup,1856) & & & & & $\mathrm{O}$ & \\
\hline Batrachoididae & & & & & & \\
\hline Porichthys porosissimus (Cuvier 1829) & $\mathrm{LF}$ & LF & LF & $\mathrm{LF}$ & $\mathrm{F}$ & $\mathrm{F}$ \\
\hline Ogcocephalidae & & & & & & \\
\hline Ogcocephalus vespertilio (Linnaeus, 1758) & & $\mathrm{O}$ & & & & $\mathrm{O}$ \\
\hline
\end{tabular}


Table 6. Continued.

\begin{tabular}{|c|c|c|c|c|c|c|}
\hline Family/Species & Barra do Sul & Penha & Itajaí & Tijucas & Porto Belo & Palhoça \\
\hline \multicolumn{7}{|l|}{ Belonidae } \\
\hline Strongylura timиси (Walbaum, 1792) & & $\mathrm{O}$ & $\mathrm{O}$ & & & \\
\hline \multicolumn{7}{|l|}{ Syngnathidae } \\
\hline Hippocampus erectus Perry, 1810 & & $\mathrm{O}$ & & & & \\
\hline Hippocampus reidi Ginsburg, 1933 & & $\mathrm{O}$ & & & & \\
\hline Microphis lineatus (Kaup, 1856) & & $\mathrm{O}$ & $\mathrm{O}$ & & & \\
\hline Bryx dunckeri (Metzelaar, 1919) & & & & $\mathrm{O}$ & & \\
\hline \multicolumn{7}{|l|}{ Fistularidae } \\
\hline Fistularia tabacaria Linnaeus, 1758 & & $\mathrm{O}$ & $\mathrm{O}$ & $\mathrm{O}$ & & \\
\hline \multicolumn{7}{|l|}{ Dactylopteridae } \\
\hline Dactylopterus volitans (Linneus, 1758) & $\mathrm{O}$ & $\mathrm{O}$ & & $\mathrm{O}$ & $\mathrm{O}$ & $\mathrm{O}$ \\
\hline \multicolumn{7}{|l|}{ Scorpaenidae } \\
\hline Scorpaena brasiliensis Cuvier, 1829 & & & & & & $\mathrm{O}$ \\
\hline Scorpaena isthmensis (Meeke and Hildebrand, 1928) & & & & & LF & \\
\hline Scorpaena plumieri Bloch, 1789 & & $\mathrm{O}$ & & & & \\
\hline \multicolumn{7}{|l|}{ Triglidae } \\
\hline Prionotus punctatus (Bloch, 1793) & $\mathrm{LF}$ & LF & & $\mathrm{O}$ & $\mathrm{F}$ & $\mathrm{F}$ \\
\hline \multicolumn{7}{|l|}{ Centropomidae } \\
\hline Centropomus parallelus Poey, 1860 & & $\mathrm{O}$ & $\mathrm{O}$ & & & $\mathrm{O}$ \\
\hline Centropomus undecimalis (Bloch, 1792) & $\mathrm{O}$ & & $\mathrm{O}$ & & & \\
\hline \multicolumn{7}{|l|}{ Serranidae } \\
\hline Diplectrum formosum (Linnaeus, 1766) & $\mathrm{O}$ & $\mathrm{O}$ & $\mathrm{O}$ & & & $\mathrm{O}$ \\
\hline Diplectrum radiale (Quoy and Gaimard, 1824) & $\mathrm{O}$ & $\mathrm{O}$ & $\mathrm{O}$ & $\mathrm{O}$ & $\mathrm{O}$ & LF \\
\hline Dules auriga Cuvier, 1829 & & $\mathrm{O}$ & $\mathrm{O}$ & & LF & LF \\
\hline Epinephelus niveatus (Valenciennes, 1828) & & $\mathrm{O}$ & & & & \\
\hline Mycteroperca acutirostris (Valenciennes, 1828) & & $\mathrm{O}$ & $\mathrm{O}$ & & & \\
\hline Rypticus randalli Courtenay, 1967 & $\mathrm{O}$ & & & & & $\mathrm{O}$ \\
\hline \multicolumn{7}{|l|}{ Priacanthidae } \\
\hline Priacanthus arenatus Cuvier, 1829 & & $\mathrm{O}$ & & & & $\mathrm{O}$ \\
\hline \multicolumn{7}{|l|}{ Carangidae } \\
\hline Caranx latus Agassiz 1831 & & $\mathrm{O}$ & $\mathrm{O}$ & & & $\mathrm{O}$ \\
\hline Caranx crysos (Mitchill, 1815) & $\mathrm{O}$ & $\mathrm{O}$ & $\mathrm{O}$ & & & \\
\hline Chloroscombrus chrysurus (Linnaeus, 1766) & $\mathrm{LF}$ & $\mathrm{F}$ & $\mathrm{F}$ & LF & LF & $\mathrm{O}$ \\
\hline Decapterus macarellus (Cuvier, 1833) & & & & & & $\mathrm{O}$ \\
\hline Oligoplites saliens (Bloch, 1793) & & $\mathrm{O}$ & $\mathrm{O}$ & & & \\
\hline Oligoplites saurus (Bloch and Schneider, 1801) & $\mathrm{O}$ & $\mathrm{O}$ & $\mathrm{O}$ & $\mathrm{O}$ & $\mathrm{O}$ & $\mathrm{O}$ \\
\hline Selar crumenophthalmus (Bloch, 1793) & & & & & & LF \\
\hline Selene setapinnis (Mitchill, 1815) & VF & LF & LF & $\mathrm{VF}$ & $\mathrm{F}$ & $\mathrm{F}$ \\
\hline Selene vomer (Linnaeus, 1758) & $\mathrm{F}$ & $\mathrm{O}$ & $\mathrm{O}$ & $\mathrm{LF}$ & LF & $\mathrm{O}$ \\
\hline Trachinotus carolinus (Linneaus, 1766) & & $\mathrm{O}$ & $\mathrm{O}$ & & & \\
\hline Trachinotus falcatus (Linnaeus, 1758) & $\mathrm{O}$ & LF & $\mathrm{O}$ & $\mathrm{O}$ & & $\mathrm{O}$ \\
\hline Trachurus lathami Nichols, 1920 & & $\mathrm{O}$ & & & & $\mathrm{O}$ \\
\hline \multicolumn{7}{|l|}{ Lutjanidae } \\
\hline Lutjanus cyanopterus (Cuvier, 1828) & & & $\mathrm{O}$ & & & \\
\hline \multicolumn{7}{|l|}{ Gerreidae } \\
\hline Diapterus rhombeus (Cuvier, 1829) & $\mathrm{LF}$ & $\mathrm{O}$ & $\mathrm{O}$ & $\mathrm{O}$ & $\mathrm{F}$ & $\mathrm{O}$ \\
\hline Eucinostomus argenteus Baird and Girard, 1855 & $\mathrm{LF}$ & $\mathrm{O}$ & $\mathrm{O}$ & & & $\mathrm{O}$ \\
\hline Eucinostomus gula (Quoy and Gaimard, 1824) & $\mathrm{O}$ & $\mathrm{O}$ & $\mathrm{O}$ & & LF & $\mathrm{O}$ \\
\hline Eucinostomus melanopterus (Bleeker, 1863) & & $\mathrm{O}$ & $\mathrm{O}$ & & $\mathrm{O}$ & $\mathrm{O}$ \\
\hline \multicolumn{7}{|l|}{ Haemulidae } \\
\hline Boridia grossidens Cuvier 1830 & & $\mathrm{O}$ & & & & \\
\hline Conodon nobilis (Linnaeus, 1758) & LF & $\mathrm{O}$ & LF & & & $\mathrm{O}$ \\
\hline Genyatremus luteus (Bloch, 1790) & & $\mathrm{O}$ & & & & \\
\hline Orthopristis ruber (Cuvier, 1830) & $\mathrm{O}$ & $\mathrm{O}$ & $\mathrm{O}$ & $\mathrm{O}$ & $\mathrm{O}$ & $\mathrm{O}$ \\
\hline Pomadasys corvinaeformis (Steindachner, 1868) & $\mathrm{F}$ & LF & LF & $\mathrm{O}$ & VF & $\mathrm{F}$ \\
\hline \multicolumn{7}{|l|}{ Sparidae } \\
\hline Diplodus argenteus (Valenciennes, 1830) & & & & & & $\mathrm{O}$ \\
\hline
\end{tabular}


Table 6. Continued.

\begin{tabular}{|c|c|c|c|c|c|c|}
\hline Family/Species & Barra do Sul & Penha & Itajaí & Tijucas & Porto Belo & Palhoça \\
\hline \multicolumn{7}{|l|}{ Polynemidae } \\
\hline Polydactylus virginicus (Linnaeus, 1758) & $\mathrm{LF}$ & & & $\mathrm{O}$ & $\mathrm{F}$ & \\
\hline \multicolumn{7}{|l|}{ Sciaenidae } \\
\hline Bairdiella ronchus (Curvier, 1830) & & $\mathrm{O}$ & LF & & & $\mathrm{O}$ \\
\hline Ctenosciaena gracilicirrhus (Metzelaar, 1919) & VF & $\mathrm{F}$ & & LF & $\mathrm{F}$ & LF \\
\hline Cynoscion jamaicensis (Vaillant and Bocourt, 1883) & $\mathrm{F}$ & LF & $\mathrm{O}$ & $\mathrm{O}$ & VF & $\mathrm{O}$ \\
\hline Cynoscion leiarchus (Cuvier, 1830) & $\mathrm{O}$ & $\mathrm{O}$ & $\mathrm{O}$ & & & \\
\hline Cynoscion striatus (Cuvier, 1829) & LF & LF & & $\mathrm{O}$ & & VF \\
\hline Isopisthus parvipinnis (Cuvier, 1830) & VF & $\mathrm{VF}$ & VF & VF & VF & LF \\
\hline Larimus breviceps Cuvier, 1830 & VF & $\mathrm{F}$ & $\mathrm{F}$ & $\mathrm{LF}$ & VF & LF \\
\hline Macrodon atricauda (Günther, 1880) & $\mathrm{F}$ & $\mathrm{F}$ & & & $\mathrm{F}$ & $\mathrm{O}$ \\
\hline Menticirrhus americanus (Linnaeus, 1758) & $\mathrm{F}$ & $\mathrm{F}$ & $\mathrm{F}$ & LF & VF & VF \\
\hline Menticirrhus littoralis (Holbrook, 1847) & $\mathrm{O}$ & LF & LF & $\mathrm{O}$ & $\mathrm{F}$ & $\mathrm{F}$ \\
\hline Micropogonias furnieri (Desmarest, 1823) & $\mathrm{O}$ & $\mathrm{F}$ & VF & $\mathrm{LF}$ & VF & LF \\
\hline Paralonchurus brasiliensis (Steindachner, 1875) & VF & $\mathrm{VF}$ & $\mathrm{VF}$ & $\mathrm{VF}$ & VF & VF \\
\hline Stellifer brasiliensis (Schultz, 1945) & VF & $\mathrm{VF}$ & VF & VF & VF & VF \\
\hline Stellifer rastrifer (Jordan, 1889) & VF & $\mathrm{VF}$ & VF & VF & VF & $\mathrm{VF}$ \\
\hline Stellifer spp. & $\mathrm{F}$ & $\mathrm{F}$ & $\mathrm{LF}$ & LF & $\mathrm{VF}$ & $\mathrm{LF}$ \\
\hline Stellifer stellifer (Bloch, 1790) & $\mathrm{F}$ & $\mathrm{F}$ & $\mathrm{F}$ & $\mathrm{F}$ & $\mathrm{F}$ & $\mathrm{F}$ \\
\hline \multicolumn{7}{|l|}{ Mullidae } \\
\hline Mullus argentinae Hubbs and Marini, 1933 & $\mathrm{O}$ & $\mathrm{O}$ & & & $\mathrm{O}$ & LF \\
\hline Upeneus parvus Poey, 1852 & & & & & & $\mathrm{O}$ \\
\hline \multicolumn{7}{|l|}{ Pomacanthidae } \\
\hline Pomacanthus paru (Bloch, 1787) & $\mathrm{O}$ & $\mathrm{O}$ & & $\mathrm{O}$ & $\mathrm{O}$ & $\mathrm{O}$ \\
\hline \multicolumn{7}{|l|}{ Labridae } \\
\hline Xyrichtys novacula (Linnaeus, 1758) & $\mathrm{O}$ & $\mathrm{O}$ & & & & \\
\hline \multicolumn{7}{|l|}{ Uranoscopidae } \\
\hline Astroscopus sexspinosus (Steindachner, 1876) & & $\mathrm{O}$ & & & & \\
\hline \multicolumn{7}{|l|}{ Blenniidae } \\
\hline Hypleurophilus fissicornis (Quoy and Gaimard, 1824) & & & $\mathrm{O}$ & & & \\
\hline \multicolumn{7}{|l|}{ Gobiidae } \\
\hline Bathygobius soporator (Valenciennes, 1837) & & & $\mathrm{O}$ & & & \\
\hline \multicolumn{7}{|l|}{ Ephippidae } \\
\hline Chaetodipterus faber (Broussonet, 1782) & $\mathrm{O}$ & $\mathrm{O}$ & $\mathrm{O}$ & & $\mathrm{LF}$ & $\mathrm{O}$ \\
\hline \multicolumn{7}{|l|}{ Trichiuridae } \\
\hline Trichiurus lepturus Linnaeus, 1758 & $\mathrm{~F}$ & LF & $\mathrm{F}$ & $\mathrm{F}$ & $\mathrm{VF}$ & LF \\
\hline \multicolumn{7}{|l|}{ Scombridae } \\
\hline Scomberomorus brasiliensis Collette, Russo and Zavala-Camin,1978 & & $\mathrm{O}$ & $\mathrm{O}$ & & & \\
\hline \multicolumn{7}{|l|}{ Stromateidae } \\
\hline Peprilus paru (Linnaeus, 1758) & LF & LF & LF & LF & $\mathrm{LF}$ & $\mathrm{F}$ \\
\hline \multicolumn{7}{|l|}{ Paralichthyidae } \\
\hline Citharichthys arenaceus Evermann and Marsh, 1900 & & & $\mathrm{O}$ & & & $\mathrm{O}$ \\
\hline Citharichthys macrops Dresel, 1885 & LF & & & & & \\
\hline Citharichthys spilopterus Günther, 1862 & LF & $\mathrm{O}$ & $\mathrm{F}$ & $\mathrm{F}$ & $\mathrm{F}$ & $\mathrm{O}$ \\
\hline Cyclopsetta chittendeni Bean, 1895 & & & & & $\mathrm{O}$ & \\
\hline Cyclopsetta decussata Gunter, 1946 & $\mathrm{O}$ & & & & $\mathrm{O}$ & \\
\hline Etropus crossotus (Jordan and Gilbert, 1881) & $\mathrm{LF}$ & LF & $\mathrm{F}$ & $\mathrm{O}$ & VF & $\mathrm{O}$ \\
\hline Etropus longimanus Norman, 1933 & $\mathrm{O}$ & $\mathrm{O}$ & & & $\mathrm{O}$ & LF \\
\hline Paralichthys brasiliensis (Ranzani, 1842) & & $\mathrm{O}$ & & & & $\mathrm{O}$ \\
\hline Paralichthys orbignyanus (Valenciennes, 1842) & & & & & $\mathrm{O}$ & $\mathrm{O}$ \\
\hline Paralichthys patagonicus Jordan, 1889 & & $\mathrm{O}$ & & & & LF \\
\hline Syacium micrurum Ranzani, 1842 & $\mathrm{~F}$ & & & & & \\
\hline Syacium papillosum (Linnaeus, 1758) & $\mathrm{F}$ & $\mathrm{O}$ & & & $\mathrm{O}$ & \\
\hline \multicolumn{7}{|l|}{ Pleuronectidae } \\
\hline Oncopterus darwinii Steindachner, 1874 & & $\mathrm{O}$ & & & & $\mathrm{O}$ \\
\hline \multicolumn{7}{|l|}{ Achiridae } \\
\hline Achirus declivis Chabanaud, 1940 & $\mathrm{LF}$ & $\mathrm{F}$ & $\mathrm{LF}$ & $\mathrm{O}$ & $\mathrm{F}$ & \\
\hline
\end{tabular}


Branco, J.O. et al.

Table 6. Continued.

\begin{tabular}{|c|c|c|c|c|c|c|}
\hline Family/Species & Barra do Sul & Penha & Itajaí & Tijucas & Porto Belo & Palhoça \\
\hline Achirus lineatus (Linnaeus, 1758) & $\mathrm{LF}$ & $\mathrm{F}$ & LF & LF & VF & $\mathrm{O}$ \\
\hline Catathyridium garmani (Jordan, 1889) & $\mathrm{O}$ & $\mathrm{O}$ & & & & $\mathrm{O}$ \\
\hline Gymnachirus nudus Kaup, 1858 & & $\mathrm{O}$ & & & & LF \\
\hline \multicolumn{7}{|l|}{ Cynoglossidae } \\
\hline Symphurus plagusia (Bloch and Schneider, 1801) & $\mathrm{LF}$ & $\mathrm{F}$ & & & & \\
\hline Symphurus tesselatus (Linnaeus, 1766) & $\mathrm{F}$ & VF & & $\mathrm{F}$ & VF & $\mathrm{F}$ \\
\hline \multicolumn{7}{|l|}{ Balistidae } \\
\hline Balistes capriscus Gmelin, 1789 & $\mathrm{O}$ & & & & & $\mathrm{O}$ \\
\hline Balistes vetula Linnaeus, 1758 & & $\mathrm{O}$ & & & & $\mathrm{O}$ \\
\hline \multicolumn{7}{|l|}{ Monocanthidae } \\
\hline Stephanolepis hispidus (Linnaeus, 1766) & $\mathrm{F}$ & $\mathrm{O}$ & $\mathrm{LF}$ & LF & $\mathrm{F}$ & $\mathrm{VF}$ \\
\hline \multicolumn{7}{|l|}{ Tetraodontidae } \\
\hline Lagocephalus laevigatus (Linnaeus, 1766) & $\mathrm{F}$ & $\mathrm{F}$ & $\mathrm{F}$ & $\mathrm{F}$ & $\mathrm{F}$ & $\mathrm{F}$ \\
\hline Sphoeroides greeleyi Gilbert, 1900 & $\mathrm{LF}$ & $\mathrm{LF}$ & $\mathrm{O}$ & LF & LF & LF \\
\hline Sphoeroides spengleri (Bloch, 1785) & $\mathrm{LF}$ & $\mathrm{LF}$ & $\mathrm{O}$ & $\mathrm{O}$ & & $\mathrm{O}$ \\
\hline Sphoeroides testudineus (Linnaeus, 1758) & $\mathrm{O}$ & $\mathrm{LF}$ & $\mathrm{O}$ & $\mathrm{O}$ & LF & $\mathrm{O}$ \\
\hline \multicolumn{7}{|l|}{ Diodontidae } \\
\hline Chilomycterus spinosus (Linnaeus, 1758) & $\mathrm{LF}$ & $\mathrm{LF}$ & $\mathrm{LF}$ & $\mathrm{O}$ & $\mathrm{O}$ & $\mathrm{O}$ \\
\hline
\end{tabular}

of Paralichthyidae (Table 6). In Carangidae, the Atlantic bumper Chloroscombrus chrysurus and the Atlantic moonfish Selene setapinis were the most frequent in trawls. In Paralichthyidae, the flatfish Citharichthys spilopterus and Etropus crossotus, as well as Syacium micrurrum and Syacium papillosum, predominated, the latter being frequent only in the region of Barra do Sul (Table 6).

Nineteen other species of fishes along the areas of artisanal fisheries of Santa Catarina present high frequencies (Table 6).

\section{Discussion}

A sustainable use of marine resources cannot be properly established without a proper dimensioning of the marine biodiversity. Knowledge and monitoring of biodiversity of an ecosystem permit not only predictions of natural effects, but also of human influences that may affect the equilibrium and distribution of species (Hendrickx et al. 2002, Lanari \& Coutinho Jr 2010).

In the present study, the richness of the seabob fishing area is represented by 216 species. Of these, only 31 (four cnidarians, three mollusks, nine crustaceans, one echinoderm and 14 fish) occurred frequently throughout captures, and may be characterized as typical of the bycatch and sharing the same environment used by the seabob shrimp.

Bycatch studies of the seabob shrimp artisanal fisheries in São Paulo (Graça-Lopes et al. 2002) and of two fishing areas in Espírito Santo (Pinheiro \& Martins 2009), identified 83 and 77 species, respectively, a smaller number than that obtained for the littoral zone of Santa Catarina. Environmental heterogeneity and differences in sampling efforts and methods, including the long temporal span in the present study, may have contributed to the larger number of species obtained herein.

Macromedusae are organisms of great ecological, medical and economic importance (Morandini et al. 2005), having a relevant role in marine food chains, when competing for food or predating eggs and larvae of commercial species (Purcell \& Arai 2001). Furthermore, they may interfere negatively in fishing activities (Nagata et al. 2009), and may cause serious accidents to swimmers and divers (Haddad Jr et al. 2002). The species Rhacostoma atlantica and Lychnorhiza lucerna were frequent in our samples. The latter species has often been associated with losses in trawling activities (Nagata et al. 2009). It is considered to load nets, making them heavier, inducing higher fuel consumption, and obliging fishermen to utilize other regions, as well as reducing the time of trawling and the capture of shrimp. Although very frequent in trawls, Chiropsalmus quadrumanus contributed with a small number of specimens, but has been associated with several accidents with fisherman, producing cases of highly painful burning (Haddad Jr et al. 2002, Nagata et al. 2009).

The region of Armação do Itapocoroy (Penha) presented the largest richness of molluscs, represented mainly by living gastropods and bivalves, which were common in samples of 1996 and 1997, when trawlings were frequently conducted over bottoms covered with shells and biodetritus. Generally, the gastropods Buccinanops gradatum and Olivancillaria urceus contributed with the largest observed abundances in trawls, similarly to that recorded for the coast of São Paulo (GraçaLopes et al. 2002). The shells of B. gradatum and O. urceus are frequently occupied by the hermit crab Loxopagurus loxochelis along the coast of Brazil and Argentina (Biagi et al. 2006), as well as by epibiontic organisms such as anthozoans and bryozoans (Ayres-Peres \& Mantelatto 2010), having an important role in the ecology of benthic communities. In the sampled areas along Santa Catarina, the hermit crabs Dardanus insignis and Loxopagurus loxochelis commonly occupied the shells of these two gastropods, which were also associated with the epibiont anemone Bunodosoma caissarum.

The squids Lolliguncula brevis, Loligo sanpaulensis and $L$. plei was also observed to have high frequencies of occurrence, particularly Lolliguncula brevis, a small, euryhaline coastal species, which is abundant in bays and estuaries (Coelho et al. 2010), although with small numerical representation in the fisheries of the seabob shrimp.

The presence of decapod crustaceans in the composition of the associate fauna is high, largely superseding the observed biomass of shrimp in conditions of being commercialized 
(Coelho et al. 1986). Decapods are one of the main groups identified in the bycatch of the seabob shrimp, being only superseded by fish (Graça-Lopes et al. 2002, Branco \& Verani 2006, Gomes \& Chaves 2006, Bernardes Jr et al. 2011, Freitas et al. 2011). In the region of Santa Catarina, families Penaeidae and Portunidae contributed with the highest observed abundances, biomasses and frequencies in the bycatch of the seabob shrimp, which has also been observed along the Brazilian littoral zone (Sampaio \& Fausto-Filho 1984, Branco \& Lunardon-Branco 1993, Mantelatto \& Fransozo 2000, Severino-Rodrigues et al. 2002, Branco \& Fracasso 2004). Among the shrimps of commercial importance, the Argentine stiletto shrimp Artemesia longinaris and the Argentine red shrimp Pleoticus muelleri, occur frequently or very frequently in samples, sharing the same environment as the seabob shrimp, as also found by Severino-Rodrigues et al. (2002) along the coast of São Paulo. However, according to Fransozo et al. (2004) and Costa et al. (2004), these two species occurred in higher abundances in deeper and more saline regions than those inhabited by the seabob shrimp. In interviews with artisanal fishermen from Itajaí (non-published data), we verified that along the year the seabob fishery vessels progress to deeper waters between $20-25 \mathrm{~m}$ in the months from September to November. They pursued the Argentine red shrimp, when the profitability of $X$. kroyeri became unsatisfactory, due to low abundances of this resource (Branco 2005). Acetes americanus and Sicyonia dorsalis were the most frequent shrimp species without commercial value, as also observed by Severino-Rodrigues et al. (2002).

For Brachyura, the blue-crabs Callinectes ornatus and C. danae are well represented in the economy and feeding of the riverside populations of Santa Catarina (Branco \& Masunari 2000). The fishery of swimming crabs represents one of the oldest activities along the Brazilian littoral zone, presently supporting several communities that live from their commercialization (Barreto et al. 2006).

The Echinodermata are important in benthic communities, occupying diverse ecological niches. Nineteen species belonging to the classes Asteroidea, Echinoidea and Holothuroidea have been included in a list of threatened species along the Brazilian coast (MMA 2014, Amaral \& Jablonski 2005, Barros Lima \& Fernandes 2009). Six of these species, Astropecten marginatus, Astropecten brasiliensis, Luidia clathrata, Luidia senegalensis, Asterina stellifera, and Echinaster brasiliensis were collected in the present study. Only $A$. marginatus was captured very frequently in trawls, indicating that artisanal fishing may be exerting pressure on the population of this species, contributing to its present extinction status.

As in other fishing regions in the Atlantic, fishes represented the main component of the bycatch of the seabob shrimp fisheries (Pinheiro \& Martins 2009, Paiva-Filho \& Schmiegelow 1986, Graça-Lopes et al. 2002, Gomes \& Chaves 2006, Schwarz Jr et al. 2007, Branco \& Verani 2006, Freitas et al. 2011). Of the fish species captured along the coast of Santa Catarina, elasmobranchs contribute a small percentage of individuals, most being occasional in samples. Young elasmobranchs captured by artisanal fishing are infrequent in our sampling. This may be due to the fact that most species evade the nets due to the low power of many fishing vessels in the area. Notwithstanding, even occasional trappings may significantly affect the recruitment capacity of species (Costa \& Chaves 2006). Considering the sharing of fishing areas by artisanal communities, and the performance of the industrial fleet, the presence of young individuals of Rhinobatos horkelii and Squatina guggenheim, threatened with extinction (MMA 2014), remains a worrying aspect for the conservation of the populations belonging to these species. The application of regulating acts becomes urgent for the management of the fishing activities considered herein.

Among bony fish, the Sciaenidae were most representative in number, biomass, frequency of occurrence and diversity of species, a fact observed also in other localities of the Atlantic Ocean, both in the south (Branco 2005, Branco \& Verani 2006, Giannini \& Paiva-Filho 1990), and in North America and the Gulf of Mexico (Pellegrin Jr 1983). According to Souza et al. (2008), it is possible that the Sciaenidae seek the same depths and areas with muddy or sandy sediments as the seabob shrimp. It may also be possible that this co-occurrence is a feeding requirement, considering the several species of shrimp found in their stomachs. The predation of shrimps by the Sciaenidae, particularly of their larval stages, may attain intensities up to three times the depletion rate exerted by the fishing fleet (Dall et al. 1990). The dominance of sciaenid species represents a key character, determining the structure of the local ichthyofaunal community. Their absence would result in a substantially different fish fauna (Freitas et al. 2011).

The occurrence of the flatfish Cyclopsetta chittendeni in the present study suggests an extension of its distributional range, which was previously known only to reach Guarujá, State of São Paulo, southeastern Brazil. The capture of three specimens of Cyclopsetta decussata represents the first record of the species for the Brazilian coast, a single specimen having been found previously only in the Gulf of Mexico (Gunter 1946). These two new occurrences reinforce the importance of continuing faunistic inventories for the knowledge and conservation of the biodiversity of the Brazilian coast. The king weakfish Macrodon ancylodon was revised by CarvalhoFilho et al. (2010). Macrodon atricauda remains valid for specimens captured in the southeast-south regions.

Thirty-one species sharing the same habitat as the seabob shrimp in Santa Catarina are mostly discarded by the artisanal fishing communities. This represents both an environmental loss, because they represent key species in the community structure, and an economic loss, because some species have potential commercial value, but are not exploited due to their small size, making their conservation on board and their future processing impractical.

Bycatch is one of the most pressing and controversial aspects of shrimp fishing. Much of the management attention associated with shrimp fisheries is focused on reducing it. Bycatch management is defined as intervention to reduce or make better use of bycatch, in order to reduce waste and threats to vulnerable and endangered species (Gillett 2008).

Alternatives for the problem of the accidental capture of the bycatch have been largely discussed worldwide, with positive results obtained from the development of Bycatch Reduction Devices - BRDs (Hannah \& Jones 2000, Broadhurst 2000, Pascoe \& Revill 2004, Eayrs 2007). Techniques to reduce bycatch levels include: 1) traditional net selectivity; 2) fishing gear development; 3) trying to take advantage of differential species behavior; and 4) time/area restrictions. Emerging ideas include:1) effort reduction; 2) incentive programs; and 3) moving the responsibility for bycatch reduction to the individual vessel level (Alverson et al. 1994). Complementary 
strategies are the creation of exclusive fishing zones, in which actions aim to ensure minimum pools of species that may recolonize adjacent areas to those subject to fishing activities. In this way, more successful conservation of species is attempted. Despite existing enforced law and several protected areas in Brazil, conservation of marine biodiversity is still broadly inadequate (Amaral \& Jablonsky 2005). Protection areas are still insufficient considering the large extent of the Brazilian coastal zone.

Bycatch is one of the most pressing and controversial aspects of shrimp fishing. Much of the management attention associated with shrimp fisheries is focused on reducing it. Conservation actions aim to ensure minimum pools of species that may recolonize areas adjacent to those subject to fishing activities. In this way, more successful conservation of species is attempted. Despite existing enforced law and several protected areas in Brazil, conservation of marine biodiversity is still broadly inadequate in our country.

In the State of Santa Catarina, small-scale shrimp fisheries is a traditional activity and dominates the local fishing economy. By inventorying the accidental bycatch, we provide a preliminary step towards defining intervention measures to reduce or make better use of this bycatch in seabob artisanal fisheries. We have attempted to identify additional threats to vulnerable and endangered species resulting from shrimp trawling.

\section{References}

ALHO, C.J.R. 2008. The value of biodiversity. Braz. J. Biol. 68 (Suppl.):1115-1118. http://www.scielo.br/pdf/bjb/v68n4s0/a18v684s.pdf

ALVERSON, D.L., FREEBERG, M.H., POPE, J.G. \& MURAWSKI, S.A. 1994. A global assessment of fisheries bycatch and discards. FAO Fish. Techn. Pap. 339:1-233.

AMARAL, A.C.Z. \& JABLONSKI, D. 2005. Conservação da biodiversidade marinha e costeira no Brasil. Megadiversidade 1:44-51. http://www.conservation.org.br/publicacoes/megadiversidade/ 08_Amaral_Jablonski.pdf

AYRES-PERES, L. \& MANTELATTO, F.L.M. 2010. Epibiont occurrence on gastropod shells used by hermit crab Loxopagurus loxochelis (Anomura: Diogenidae) on the northern coast of São Paulo, Brazil. Zoologia 27:222-227. http://www.scielo.br/pdf/ $\mathrm{zool} / \mathrm{v} 27 \mathrm{n} 2 / 10 . \mathrm{pdf}$

BARRETO, A.V., LEITE, L.M.A.B. \& AGUIAR, M.C.A. 2006. Maturidade sexual das fêmeas de Callinectes danae (Crustacea, Decapoda, Portunidae) nos estuários dos rios Botafogo e Carrapicho, Itamaracá-PE Brasil. Iheringia Sér. Zool. 96:141-146. http://www.scielo.br/pdf/isz/v96n2/a03v96n2.pdf

BARROS LIMA, E.J. \& FERNANDES, M.L.B. 2009. Diversidade de equinodermos (Echinodermata) no Estado de Pernambuco (Brasil). Rev. Bras. Zoociênc. 11:55-63. http://zoociencias.ufjf. emnuvens.com.br/zoociencias/article/view/448/418

BERNARDES JR, J.J., RODRIGUES FILHO, J.L., BRANCO, J.O \& VERANI, J.R. 2011. Spatiotemporal variations of the ichthyofauna structure accompanying the seabob shrimp Xiphopenaeus kroyeri (Crustacea: Penaeidae) fishery, in important fisheries areas of the Santa Catarina shore, Brazil. Zoologia 28:151-164. http:// www.scielo.br/pdf/zool/v28n2/a02v28n2.pdf

BIAGI, R., MEIRELES, L.A., SCELZO, M.A. \& MANTELATTO, F.L.M. 2006. Comparative study of shell choice by the southern endemic hermit crab Loxopagurus loxochelis from Brazil and Argentina. Rev. Chil. Hist. Nat. 79:481-487. http://www.scielo.cl/ pdf/rchnat/v79n4/art07.pdf

BRANCO, J.O. 2005. Biologia e pesca do camarão sete-barbas Xiphopenaeus kroyeri (Heller, 1862) (Crustacea, Penaeidae), na Armação do Itapocoroy, Penha, Santa Catarina, Brasil. Rev. Bras. Zool. 22:1050-1062. http://www.scielo.br/pdf/rbzool/v22n4/a34v22n4.pdf

BRANCO, J.O. \& FRACASSO, H.A. 2004. Ocorrência e abundância da carcinofauna acompanhante na pesca do camarão sete-barbas, Xiphopenaeus kroyeri Heller (Crustacea, Decapoda), na Armação do Itapocoroy, Penha, Santa Catarina, Brasil. Rev. Bras. Zool. 2:95-301. http://www.scielo.br/pdf/rbzool/v21n1/19716.pdf

BRANCO, J.O. \& LUNARDON-BRANCO, M.J. 1993. Aspectos da biologia de Callinectes ornatus Ordway, 1863 (Decapoda, Portunidae) da região de Matinhos, Paraná, Brazil. Arq. Biol. Tecn. 36:489-496.

BRANCO, J.O. \& MASUNARI, M, 2000. Reproductive ecology of the blue crab, Callinectes danae Smith, 1869 in the Conceição Lagoon system, Santa Catarina Isle, Brazil. Rev. Bras. Biol. 60: 17-27.

BRANCO, J.O. \& VERANI, J.R. 2006. Análise quali-quantitativa da ictiofauna acompanhante na pesca do camarão sete-barbas, na Armação do Itapocoroy, Penha, Santa Catarina. Rev. Bras. Zool. 23:381-391. http://www.scielo.br/pdf/rbzool/v23n2/a11v23n2.pdf

BROADHURST, M.K. 2000. Modifications to reduce bycatch in prawn trawls: A review and framework for development. Rev. Fish. Biol. Fish. 10:27-60. http://download.springer.com/static/pdf/ 39/art $\% 253 \mathrm{~A} 10.1023 \% 252 \mathrm{FA} \% 253 \mathrm{~A} 1008936820089$.pdf?auth66 = 1411041824_029b5172017cc8206c3d3cf383b3b267\&ext =.pdf

CARVALHO-FILHO, A., SANTOS, S. \& SAMPAIO, I. 2010. Macrodon atricauda (Günther, 1880) (Perciformes: Sciaenidae), a valid species from the southwestern Atlantic, with comments on its conservation. Zootaxa 2519:48-58. http://www.mapress.com/ zootaxa/2010/f/zt02519p058.pdf

COELHO, J.A.P., PUZZI, A., GRAÇA-LOPES, R., RODRIGUES, E.S. \& PRETO JR, O. 1986. Análise da rejeição de peixes na pesca artisanal dirigida ao camarão sete-barbas (Xiphopenaeus kroyeri) no litoral do estado de São Paulo. Bol. Inst. Pesca 13:51-61. ftp://ftp.sp.gov.br/ftppesca/13_2_51-61.pdf

COELHO, L.I., MUTO, E.Y., MARIAN, J.E.A.R. \& SOARES, L.S. H. 2010. Contribuição ao conhecimento da dieta, atividade alimentar e reprodução de Lolliguncula brevis (Blainville, 1823) na região costeira de Santos (Estado de São Paulo). Bol. Inst. Pesca 36:225-236. ftp://ftp.sp.gov.br/ftppesca/36_3_225-236.pdf

COSTA, R.C., FRANSOZO, A. \& PINHEIRO, A.P. 2004. Ecological distribution of the shrimp Pleoticus muelleri (Bate, 1888) (Decapoda: Penaeoidea) in southeastern Brazil. Hydrobiologia 529:195203. http://download.springer.com/static/pdf/428/art $\% 253 \mathrm{~A} 10.1007 \%$ 252Fs10750-004-6410-x.pdf?auth66 = 1411042402_97506bd791f174e6b 02cefd13ad5610a\&ext $=$.pdf

COSTA, L. \& CHAVES, P.T.C. 2006. Elasmobrânquios capturados pela pesca artisanal na costa sul do Paraná e norte de Santa Catarina, Brasil. Biota Neotropica 6(3):1-10. http://www. biotaneotropica.org. br/v6n3/pt/abstract?article + bn02706032006.

DALL, W., HIL, B.J., ROTHLISBERG, P.C. \& STAPLES, D.J. 1990. The biology of the Penaeidae. In Advances in Marine Biology, Volume 27 (Blaxter, J.H.S. \& Southward, A.J., eds). Academic Press, San Diego.

EAYRS, S. 2007. A guide to bycatch reduction in tropical shrimp-trawl fisheries. FAO, Rome. http://www.fao.org/docrep/015/a1008e/ a1008e.pdf

FRANSOZO, A., COSTA, R.C., CASTILHO, A.L. \& MANTELATTO, F.L.M. 2004. Ecological distribution of the shrimp "camarão serrinha" Artemesia longinaris (Decapoda, Penaeidae) in Fortaleza Bay, Ubatuba, Brazil, in relation to abiotic factors. Rev.Invest. Desar. Pesq. 16:45-53. http://www.oceandocs.org/ bitstream/1834/1537/1/Rev\%20Invest $\% 20$ Desarr $\% 20$ Pesq $\% 2016 \%$ 2043-50.pdf

FREITAS M.O., SPACH, H.L. \& HOSTIM-SILVA, M. 2011 Variação espaço-temporal da assembléia de peixes demersais em uma área de pesca do camarão sete-barbas no sul do Brasil. Neotrop. Biol. Cons. 6:44-54. file:///C:/Users/cliente/Downloads/996-2862-1-PB. pdf 
GIANNINI, R. \& PAIVA-FILHO, A.M. 1990. Os Sciaenidae (Teleostei: Perciformes) da Baía de Santos (SP), Brasil. Bol. Inst. Oceanogr. 38:69-86. http://www.scielo.br/pdf/bioce/v38n1/v38n1a07.pdf

GILLETT, R. 2008. Global study of shrimp fisheries. FAO Fish. Techn Pap 475:1-331. http://www.fao.org/docrep/013/i0300s/10300s.pdf

GOMES, I.D. \& CHAVES, P.T. 2006. Ictiofauna integrante da pesca de arrasto camaroeiro no litoral sul do estado do Paraná, Brasil. Bioikos 20:9-13. file:///C:/Users/cliente/Downloads/10183-18881-1PB.pdf

GRAÇA-LOPES, R., TOMÁS, A.R.G., TUTUI, S.L.S., SEVERINORODRIGUES, E. \& PUZZI, A. 2002. Comparação da dinâmica de desembarques de frotas camaroeiras do Estado de São Paulo, Brasil. Bol. Inst. Pesca 28:163-171. ftp://ftp.sp.gov.br/ftppesca/ 28_2_163-171.pdf

GRAY, J.S. 1997. Marine biodiversity: patterns, threats and conservation needs. Biod. Cons. 6:153-175. http://download.springer. com/static/pdf/975/art\%253A10.1023\%252FA\%253A1018335901847. pdf?auth66 $=$ 1411044141_17f2ccc0d1246accff87c9183dc39c87\&ext $=$. pdf

GUNTER, G. 1946. A new species of flatfish, Cyclopsetta decussata (Pleuronectidae), from the Texas coast. Copeia 1:27-28.

HADDAD JR, V., SILVEIRA, F.L., CARDOSO, J.L.C. \& MORANDINI, A.C. 2002. A report of 49 cases of cnidarian envenoming from southeastern Brazilian coastal waters. Toxicon 40:1445-1450.

HANNAH, R.W. \& JONES, S. 2000. By-catch reduction in an ocean shrimp trawl from a simple modification to the trawl footrope. J. Northw. Atl. Fish Sci. 27:227-233. http://journal.nafo.int/J27/ hannah.pdf?origin = publication_detail

HENDRICKX, E., BRUSCA, R.C. \& RESÉNDIZ, G.R. 2002. Biodiversity of macro crustaceans in the Gulf of California, Mexico. Contr. Stud. East Pac. Crust. 1:349-367. https://www.desertmuseum.org/center/seaofcortez/Hendirckx_et_al._2002.pdf

KEUNECKE, K.A., VIANNA, M., FONSECA, D.B.F., D' INCAO, F. 2007. The pink-shrimp trawling bycatch in the northern coast of São Paulo, Brazil, with emphasis on crustaceans. Nauplius 15:49-55.

LANARI, M.O., COUTINHO, R. 2010. Biodiversidade e funcionamento de ecossistemas: Síntese de um paradigma e sua expansão em ambientes marinhos. Oecol. Austr. 14:959-988.

MANTELATTO, F.L.M. \& FRANSOZO, A. 2000. Brachyuran community in Ubatuba Bay, northern coast of São Paulo State, Brazil. J. Shellf. Res. 19:701-709.

MMA - Ministério Do Meio Ambiente. 2014. Lista das espécies de fauna ameaçadas de extinção. http://www.icmbio.gov.br/portal/ biodiversidade/fauna-brasileira/lista-de-especies.html

MORANDINI, A.C., ASCHER, D., STAMPAR, S.N. \& FERREIRA, J. F.V. 2005. Cubozoa e Scyphozoa (Cnidaria: Medusozoa) de águas costeiras do Brasil. Ilheringia Sér. Zool. 95:281-294. http://www.scielo. br/pdf/isz/v95n3/26542.pdf

NAGATA, R.M., HADDAD, M.A. \& NOGUEIRA JR, M. 2009. The nuisance of medusae (Cnidaria, Medusozoa) to shrimp trawls in central part of southern Brazilian Bight, from the perspective of artisanal fishermen. Pan-Amer. J. Aquat. Sci. 4:312-325.

PAIVA-FILHO, A.M. \& SCHMIEGELOW, J.M.M. 1986. Estudo sobre a ictiofauna acompanhante da pesca do camarão sete-barbas (Xiphopenaeus kroyeri) nas proximidades da Baía de Santos - SP. I. Aspectos quantitativos. Bol. Inst. Oceanogr S. Paulo 34:79-85. http://www.scielo.br/pdf/bioce/v34/v34a07.pdf

PASCOE, S. \& REVILL, A. 2004. Costs and benefits of bycatch reduction devices in European brown shrimp trawl fisheries. Env. Res. Econ. 27:43-64. http://download.springer.com/static/pdf/944/ art $\% 253$ A $10.1023 \% 252 \mathrm{FB} \% 253$ AEARE.0000016794.43136.0a.pdf? auth66 $=1411045922 \_346 \mathrm{c} 075 \mathrm{f} 9 \mathrm{a} 30785202 \mathrm{c} 6 \mathrm{~b} 46 \mathrm{f} 6 \mathrm{~b} 8 \mathrm{fa} 93 \mathrm{~d} \& \mathrm{ext}=$. pdf

PELLEGRIN JR, G. 1983. Descarte de pescado en la pesquería de camaron en el sudeste de Estados Unidos. Pesca acompanante del camaron - un regalo del mar: informe de una consulta técnica sobre la utilización de la pesca acompanante del camaron celebrada em Georgetown, Guyana, 27-30 octubre 1981. Ontario, Ottawa, pp. 56-60.

PEREZ, J.A.A. 2009. Biodiversidade Marinha: Uma herança ameaçada? Ciênc. Cult. 62:42-44. http://cienciaecultura.bvs.br/pdf/ cic/v62n3/a17v62n3.pdf

PINHEIRO, H.T. \& MARTINS, A.S. 2009. Estudo comparativo da captura artisanal do camarão sete-barbas e sua fauna acompanhante em duas áreas de pesca do litoral do Espírito Santo. Bol. Inst. Pesca. 35:215-225. ftp://ftp.sp.gov.br/ftppesca/35_2_215-225. pdf

PURCELL, J.E. \& ARAI, M.N. 2001. Interactions of pelagic cnidarians and ctenophores with fish: a review. Hydrobiologia 451:27-44. http://download.springer.com/static/pdf/187/art \%253A10.1023\% 252FA\%253A1011883905394.pdf?auth66 = 1411047126_6 a89af2ae5ad3d6fe1a9a18f4291fddc\&ext = .pdf

ROBERT, R., BORZONE, C.A. \& NATIVIDADE, C.D. 2007. Os camarões da fauna acompanhante na pesca dirigida ao camarão sete-barbas (Xiphopenaeus kroyeri) no litoral do Paraná. Bol. Inst. Pesca. 33:237-246. ftp://ftp.sp.gov.br/ftppesca/33_2_237246.pdf

SAMPAIO, C.M.S. \& FAUSTO-FILHO, J. 1984. Considerações sobre a bioecologia dos crustáceos decápodes da enseada do Mucuripe (Fortaleza, Ceará Brasil). Arq. Ciênc. Mar. 23:11-24. http://www. labomar.ufc.br/images/stories/arquivos/ArqCienMar/V23_1984/ acm_1984_23_1_02.pdf

SANTOS, M.C.F. 2000. Diversidade ecológica da ictiofauna acompanhante nas pescarias de camarões em Tamandaré (PernambucoBrasil). Bol. Técn.-Cient. CEPENE 8:7-26.

SANTOS, M.C.F., ALMEIDA, L. \& SILVA, C.G.M. 2008. Avaliação quali-quantitativa da ictiofauna acompanhante na pesca do camarão sete-barbas, Xiphopenaeus kroyeri (Heller, 1862) no município de Caravelas (Bahia - Brasil). Bol. Técn.-Cien. CEPENE 16:99-107.

SCHWARZ JR, R., FRANCO, A.C.P., SPACH, H.L., SANTOS, C., PICHLER, H.A. \& QUEIROZ, G.M.L.N. 2007. Variação da estrutura espacial da ictiofauna demersal capturada com rede de arrasto de porta na Baía dos Pinheiros, PR. Bol. Inst. Pesca 33: 157-169. ftp://ftp.sp.gov.br/ftppesca/33_2_157-169.pdf

SEVERINO-RODRIGUES, E., GUERRA， D.S.F. \& GRAÇALOPES, R. 2002. Carcinofauna acompanhante da pesca dirigida ao camarão sete-barbas (Xiphopenaeus kroyeri) desembarcado na praia do Perequê, Estado de São Paulo, Brasil. Bol. Inst. Pesca 28:33-48. ftp://ftp.sp.gov.br/ftppesca/instituto $\% 20 \mathrm{de} \% 20$ pesca $\%$ 2033-48.pdf

SEVERINO-RODRIGUES, E., HEBLING, N.J. \& GRAÇA-LOPES, R. 2007. Biodiversidade no produto da pesca de arrasto-de-fundodirigida ao lagostim, Metanephrops rubellus (Moreira, 1903), desembarcado no litoral do estado de São Paulo, Brasil. Bol. Inst. Pesca 33:171-182. ftp://ftp.sp.gov.br/ftppesca/33_2_171-182.pdf

SLAVIN, J.W. 1983. Utilización de la pesca acompañante del camarón. Pesca acompañante - Un regalo del mar. CIID, Ottawa, pp. 67-71.

SOUZA, U.P., COSTA, R.C., MARTINS, I.A. \& FRANSOZO, A. 2008. Associações entre as biomassas de peixes Sciaenidae (Teleostei: Perciformes) e de camarões Penaeoidea (Decapoda: Dendrobranchiata) no litoral norte do Estado de São Paulo. Biota Neotropica 8:83-92. http://www.scielo.br/pdf/bn/v8n1/ a11v8n1.pdf

TISCHER, M. \& SANTOS, M.C.F. 2001. Algumas considerações sobre a ictiofauna acompanhante da pesca de camarões na foz do rio São Francisco (Alagoas/Sergipe-Brasil). Bol. Técn.- Cient. CEPENE 9:155-165.

TUNDISI, J.G. \& MATSUMURA-TUNDISI, T. 2008. Biodiversity in the Neotropics: ecological, economic and social values. Braz. J. Biol. 68:913-915.

VIANNA, M. 2001. Shrimp: goal or catch fish. Techn. Not. FACIMAR 5:47-49. 
Branco, J.O. et al.

VIANNA, M. \& ALMEIDA, T. 2005. Bony fish bycatch in the southern Brazil pink shrimp (Farfantepenaeus brasiliensis and F. paulensis) fishery. Braz. Arch. Biol. Techn. 48:611-623.

VIANNA, M., COSTA, F.E.S. \& FERREIRA, C.N. 2004. Lengthweight relationship of the fish caught by-catch by shrimp fishery in the southeastern coast of Brazil. Bull. Fish. Inst. S. Paulo 30:81-85.

VIANNA, M. \& D'INCAO, F. 2006. Evaluation of by-catch reduction devices for use in the artisanal pink shrimp (Farfantepenaeus paulensis) fishery in Patos Lagoon, Brazil. Fish. Res. 81:331-336.
VIANNA, M., THOMAS, S.R.G. \& VERANI, J.R. 2000. Aspects of the biology of the Atlantic Midshipman Porichthys porosissimus (Teleostei, Batrachoididae): an important by-catch species of shrimp trawling off southern Brazil. Braz. J. Oceanogr. S. Paulo 48:133-142.

VIANNA, M. \& VERANI, J.R. 2002. Biologia populacional de Orthopristis ruber (Teleostei, Haemulidae) espécie acompanhante da pesca de arrasto do camarão-rosa, no sudeste brasileiro. Atlântica 23:2-36.

WILSON, E.O. 1992. The diversity of life. Norton, New York.

Received 14/04/2015

Revised 14/04/2015

Accepted 15/04/2015 